\title{
Parkinson's disease treatments: focus on transcranial direct current stimulation (tDCS)
}

\author{
This article was published in the following Dove Press journal: \\ Journal of Parkinsonism and Restless Legs Syndrome \\ 16 October 2017 \\ Number of times this article has been viewed
}

\author{
Vassilios Tahtis' \\ Diego Kaski ${ }^{1,2}$ \\ 'Division of Brain Sciences, Imperial \\ College London, Charing Cross \\ Hospital Campus, ${ }^{2}$ Sobell Department \\ for Motor Control and Movement \\ Disorders, University College London, \\ London, UK
}

\begin{abstract}
Parkinson's disease (PD) is among the most common progressive neurodegenerative conditions worldwide, characterized by bradykinesia, rigidity, tremor, and postural instability, in addition to a variety of cognitive and behavior complications. Current pharmacological treatment options focus on dopaminergic replacement, but these become less efficacious as the disease progresses. Noninvasive brain stimulation techniques have therefore gained favor in recent years as a means of treating the motor and non-motor complications of PD. Here we review the evidence behind the use of transcranial direct current stimulation in the treatment of motor and non-motor complications of PD, discuss the limitations that have hindered the introduction of transcranial direct current stimulation into mainstream clinical practice, and highlight future directions that may enable the translation of this research tool into clinical use.
\end{abstract}

Keywords: Parkinson's disease, transcranial direct current stimulation, motor, cognitive, non-motor

\section{Introduction}

Parkinson's disease (PD) is a progressive neurodegenerative disorder characterized by resting tremor, rigidity, bradykinesia, and postural instability, in addition to non-motor complications that may predate the motor symptoms. As one of the most common human degenerative disorders, it has a major socio-economic impact worldwide. The motor complications result from loss of dopaminergic neurons in the substantia nigra of the basal ganglia, and thus pharmacological treatments focus on dopamine replacement, most commonly with levodopa, or dopamine agonists. It is a well-recognized phenomenon that treatment response diminishes as the disease progresses, alongside the development of additional symptoms from degeneration of non-dopaminergic neuronal systems. One feature of such progression is the emergence of gait and postural disturbance that is often refractory to treatment and becomes a central cause of disability. So-called non-motor complications of PD include constipation, anosmia, sexual dysfunction, and sleep abnormality.

Cognitive and behavioral deficits and psychiatric manifestations are a further hallmark of this disorder which become evident as the disease progresses and increase with the age and severity of the disease. ${ }^{1}$ These symptoms are often overlooked but may have a devastating impact on the function and quality of life of patients with $\mathrm{PD},{ }^{2}$ being notoriously difficult to treat.

It is increasingly recognized that there may be an optimal time window to consider surgical interventions - primarily deep brain stimulation (DBS) targeting a variety
Correspondence: Diego Kaski Sobell Department for Motor Control and Movement Disorders, University College London, Queen Square, WCIN 3BG, London, UK

Email d.kaski@ucl.ac.uk 
of nuclei - for motor complications of PD; during the early stages of the disease, patients typically respond well to oral medications, whereas with very advanced disease axial motor signs and psychiatric complications, they may respond less well to DBS. Thus, DBS is of particular benefit between the occurrence of levodopa-induced motor complications and the development of levodopa-resistant symptoms. DBS requires careful patient selection in light of the potential neurosurgical and anesthetic risks and in some patients can have significant neuropsychiatric sequelae. Consequently, there has been a growing need to supplement pharmacological treatments for PD with less invasive stimulation techniques that do not impose surgical risks. Therapeutic studies of non-invasive brain stimulation, including repetitive transcranial magnetic stimulation (rTMS), have yielded promising results in PD. Two meta-analyses concluded that there was a modest therapeutic effect of rTMS in motor performance in $\mathrm{PD},{ }^{3,4}$ with no safety concerns.

Transcranial direct current stimulation (tDCS) is a mode of non-invasive brain stimulation whereby a direct current is delivered to the head via surface electrodes positioned on the scalp to induce polarity-dependent changes of the underlying cortex. The use of this technique to modulate cortical excitability ${ }^{5}$ and promote motor and cognitive learning in healthy adults $^{6}$ and motor recovery in chronic stroke ${ }^{7}$ has raised interest in tDCS as an intervention in PD. While tDCS has been shown to improve motor learning and cognitive function in PD, outcomes have been mixed.

Here we review the evidence behind the use of tDCS in the treatment of motor and non-motor complications of PD, discuss the limitations that have hindered the introduction of tDCS into mainstream clinical practice, and highlight future directions that may enable the translation of this research tool into clinical use.

\section{Search strategy}

The search focused on published literature with search aggregators used to access content from key databases. OvidSp, PubMed, EBSCO Host, and Google Scholar were all used. Terms were searched in systematic, alternate combinations to optimize the depth of the search and included (with variations): Parkinson's, bradykinesia, dyskinesia, tDCS, transcranial, direct current, NIBS, non-invasive, brain stimulation, anodal, cathodal. The two authors independently read titles and abstracts to exclude papers irrelevant to the review. The focus of the search was on experimental clinical research which investigated the efficacy of tDCS in PD and any associated symptoms (motor and non-motor). Papers that met this inclusion criteria (experimental research, participants, and intervention) were retrieved in full and again checked for applicability by the two authors. Publications in English were only included, and we also searched the reference lists of any studies identified. This search process was repeated at regular intervals until June 2017. No date restriction was applied to the search. Similar to Lefaucheur's comprehensive review, ${ }^{8}$ we chose to exclude three studies that used tDCS as a priming mechanism for testing an alternative intervention, namely rTMS. ${ }^{9-11}$

\section{tDCS}

tDCS involves the delivery of a low-intensity current (1-2 $\mathrm{mA})$ using relatively large electrodes $(\sim 2-10 \mathrm{~cm})$ generally placed on the scalp. Most studies of tDCS employ a "bipolar" montage consisting of one anode and one cathode to induce an electric field that passes across the various cranial structures (skin, bone, pia, and cerebrospinal fluid) to reach the brain parenchyma. The size of the electric field over the brain parenchyma varies across studies from 0.3 to1.6 $\mathrm{V} / \mathrm{m}$ - according to head models or in vivo measurements reflecting anatomical differences between individuals, electrode size, shape, and placement. ${ }^{12-14}$ Computational modeling has offered insights into the mechanism of action of tDCS, although much work is still needed to better define the current flow across and between cerebral structures. For example, controversy abounds regarding whether the peak current densities beneath the electrodes are sufficient to modulate neuronal activity. However, experimental data do suggest that neurons are sensitive to such weak electric fields, resulting in modulation of action potential firing, synaptic plasticity, and promotion of neurogenesis, ${ }^{15-17}$ in addition to subtle effects on supporting glial cells. ${ }^{18,19}$ One of the experimental benefits of tDCS is the ability to administer a "sham" stimulation that naïve subjects cannot differentiate from "real" stimulation, allowing for the design of controlled studies. This form of placebo is usually achieved by delivering a brief ( $<30$ seconds) ramp current causing a mild subjective sensation over the scalp and then turning the stimulation off.

Recently published studies on animal models provide strong evidence for the potential of tDCS to modulate dopaminergic pathways. Frontal tDCS in rats ${ }^{20}$ and tDCS in a mouse model of $\mathrm{PD}^{21}$ led to increased dopaminergic release, resulting in an elevation of striatal dopamine levels, in some instances lasting for more than 6 hours after stimulation. In a monkey model of advanced PD, frontal anodal tDCS was shown to activate neurons in the dopaminergic substantia nigra. ${ }^{22}$

It should be noted that $\mathrm{tDCS}$ differs from other methods of cortical stimulation, such as rTMS, which are able to induce 
sufficient current intensity to directly elicit action potentials from cortical axons. ${ }^{23,24}$ Conversely, tDCS has been shown to modulate the resting membrane potential of cortical axons in the direction of depolarization or hyperpolarization, according to the polarity of the electrodes (anode vs cathode) and the orientation of the axons in the induced electric field. In its most simplistic form, anodal tDCS induces neuronal depolarization (excitability) and cathodal tDCS hyperpolarization (inhibition). Early into the phase of renewed interest in tDCS, experiments demonstrated excitability changes which persisted beyond the stimulation period. ${ }^{5}$ This post-stimulation modulation of neuronal excitability over long periods (up to 90 minutes) renders tDCS an attractive therapeutic tool in patients with PD.

Table 1 summarizes the current studies exploring the use of tDCS in patients with PD, across motor and non-motor domains. Discussion of these studies according to the motor or cognitive target is as follows.

\section{tDCS for upper limb motor function in PD}

Bradykinesia is considered to be the defining feature of PD, clinically evident as a decrement in the amplitude and velocity of repetitive movements. ${ }^{2}$ In one of the first studies of tDCS in PD, Fregni et $\mathrm{al}^{25}$ evaluated the effects of tDCS on both motor function - as assessed by Unified Parkinson's Disease Rating Scale (UPDRS) part III, simple reaction time, and Purdue Pegboard test (a test of manual dexterity) - and motor-evoked potentials (MEPs) of the hand in patients with PD. They applied anodal tDCS over the primary motor cortex (M1; $n=9)$, cathodal stimulation of M1 $(n=8)$, and anodal stimulation of dorsolateral prefrontal cortex (DLPFC, a cortical area involved primarily in cognitive processes - see tDCS for cognition section; $n=9$ ). All stimulations were compared to sham, in a double-blinded manner. A constant current of $1 \mathrm{~mA}$ intensity was applied for 20 minutes. They found that anodal stimulation of M1 was associated with a significant improvement of motor function compared to sham stimulation in the UPDRS part III $(21.9 \%$ mean improvement after active tDCS compared to $-1.6 \%$ with sham stimulation) and reaction times. Although the study was not powered to assess multiple comparisons, descriptive analysis suggested that improvements in UPDRS seemed to be driven mostly by changes in rigidity and to a less degree bradykinesia. This effect was not observed for cathodal stimulation of M1 or anodal stimulation of DLPFC, although a small (nonstatistically significant) improvement in motor function was noted. Furthermore, whereas anodal stimulation of M1 significantly increased MEP amplitude, cathodal stimulation of M1 significantly decreased the amplitude. Thus, tDCS over M1 was able to modulate neuronal excitability - manifest as increased hand MEPs - and such neuronal changes may underpin the improvement in motor function in patients with PD. The number of participants in each experiment presenting with their left or right side most-affected was almost equal; however, the authors did not consider lateralization effects (see Key considerations for a large-scale, multicenter clinical trial of tDCS in PD section).

Benninger et $\mathrm{al}^{26}$ investigated the efficacy of $\mathrm{tDCS}$ in the treatment of motor and cognitive aspects of PD using a randomized, double-blind, sham-controlled study design. Anodal tDCS (2 mA) was delivered for 20 minutes through a large electrode (surface $97.5 \mathrm{~cm}^{2}$; current density 0.021 $\mathrm{mA} / \mathrm{cm}^{2}$ ) that was placed symmetrically over either the premotor and motor (electrode center $10 \mathrm{~mm}$ anterior to $\mathrm{Cz}$ ) or the prefrontal cortices (forehead above eyebrows $147 \mathrm{~mm}$ anterior to $\mathrm{Cz}$ ). They stimulated a single target area during one session and alternated the position of the anode between sessions (starting with the motor area) so that each target area was stimulated four times. A cathode electrode $\left(25 \mathrm{~cm}^{2}\right.$ each) was positioned over each mastoid. Patients underwent eight treatment sessions over a period of 2.5 weeks, with a minimum washout period of 48 hours. Bradykinesia significantly improved with tDCS compared to sham intervention $(-28.4 \%$ vs $-11 \%, p=0.002$, when "on" and $-36.0 \%$ vs $-17.8 \%, p<0.0001$, when "off"). Although bradykinesia improved irrespective of whether patients were "on" or "off" dopaminergic medication, the rate of change was greater during "off" periods. A link between tDCS and dopamine has been investigated experimentally, with a suggestion that L-dopa can reduce the tDCS-related modulation of cortical excitability, ${ }^{27}$ in keeping with the observations in this study. However, this study employed a different tDCS montage for the sham stimulation compared to the active stimulation, raising concerns about the methodological rigor.

Doruk et $\mathrm{al}^{28}$ performed a sham-controlled, doubleblinded study across two centers, assessing the immediate and long-term effects of ten consecutive sessions of tDCS over the anode on the right DLPFC $(n=5)$, left DLPFC $(n=6)$ or sham $(n=7)$. Although the primary outcomes were cognition and mood, they evaluated the motor function. As expected, given the findings of Fregni et $\mathrm{al}^{25}$ and neuroanatomical evidence supporting a more cognitive function of DLPFC, they did not find any significant improvements on the UPDRS part III, Purdue Pegboard, or reaction times with tDCS.

Costa-Ribero et al showed no difference between primary motor cortex anodal tDCS and sham (when combined with gait training) in bradykinesia subscores of the UPDRS. ${ }^{29}$ Similarly, Schabrun et $\mathrm{al}^{30}$ combined anodal (and sham) stimulation with repeated gait training sessions and recorded 
Table I Summary of studies exploring the use of tDCS across motor and non-motor complications in Parkinson's disease

\begin{tabular}{|c|c|c|c|c|c|c|c|c|c|}
\hline Authors & Year & Motor & Cognitive & Other & $\begin{array}{l}\text { Electrode } \\
\text { size }\left(\mathrm{cm}^{2}\right)\end{array}$ & $\begin{array}{l}\text { Electrode } \\
\text { intensity } \\
(\mathrm{mA})\end{array}$ & Anode & Cathode & Sessions \\
\hline \multirow[t]{2}{*}{$\begin{array}{l}\text { Boggio } \\
\text { et } \mathrm{a}^{71}\end{array}$} & 2006 & & $\begin{array}{l}\text { Working } \\
\text { memory }\end{array}$ & & 35 & 1 & $\begin{array}{l}\text { Left DLPFC; MI } \\
\text { (left assumed) }\end{array}$ & $\begin{array}{l}\text { Contralateral } \\
\text { right orbit }\end{array}$ & 2 \\
\hline & & & & & & 2 & $\begin{array}{l}\text { Left DLPFC; MI } \\
\text { (left assumed) }\end{array}$ & $\begin{array}{l}\text { Contralateral } \\
\text { right orbit }\end{array}$ & 2 \\
\hline \multirow[t]{3}{*}{ Fregni et $\mathrm{a}^{25}$} & 2006 & UL & & & 35 & 1 & Left MI & $\begin{array}{l}\text { Right } \\
\text { supraorbital }\end{array}$ & I \\
\hline & & & & & 35 & 1 & Right supraorbital & Left MI & I \\
\hline & & & & & 35 & 1 & Left DLPFC & $\begin{array}{l}\text { Right } \\
\text { supraorbital }\end{array}$ & I \\
\hline $\begin{array}{l}\text { Benninger } \\
\text { et } \mathrm{al}^{26}\end{array}$ & 2010 & $\begin{array}{l}\text { Gait and } \\
\text { UL }\end{array}$ & $\begin{array}{l}\text { Reaction } \\
\text { time }\end{array}$ & Depression & $\begin{array}{l}97.5 \text { (anode); } \\
25 \text { (cathode) }\end{array}$ & 2 & $\begin{array}{l}10 \mathrm{~cm} \text { anterior to } \\
\mathrm{Cz} \times 4 \text { sessions; } \\
\text { prefrontal } \\
\text { cortices } \\
\text { (forehead above } \\
\text { eyebrows) } \times 4 \\
\text { sessions }\end{array}$ & Mastoids & $8(2 / 3 p w)$ \\
\hline $\begin{array}{l}\text { Pereira } \\
\text { et } \mathrm{al}^{76}\end{array}$ & 2013 & & & $\begin{array}{l}\text { Verbal } \\
\text { fluency }\end{array}$ & 35 & 2 & $\begin{array}{l}\text { Left DLPFC or } \\
\text { left TPC }\end{array}$ & $\begin{array}{l}\text { Right } \\
\text { supraorbital }\end{array}$ & $\begin{array}{l}\text { I (left } \\
\text { DLPFC) + I } \\
\text { (left TPC) }\end{array}$ \\
\hline $\begin{array}{l}\text { Verheyden } \\
\text { et } \mathrm{al}^{41}\end{array}$ & 2013 & $\begin{array}{l}\text { Gait and } \\
\text { posture }\end{array}$ & & & Not stated & I & $\begin{array}{l}\text { MI of dominant } \\
\text { hemisphere }\end{array}$ & $\begin{array}{l}\text { Contralateral } \\
\text { supraorbital }\end{array}$ & I + sham \\
\hline Doruk et $\mathrm{al}^{28}$ & 2014 & UL & Exec & $\begin{array}{l}\text { Mood/ } \\
\text { behavior }\end{array}$ & 35 & 2 & $\begin{array}{l}\text { Left or right } \\
\text { DLPFC }\end{array}$ & $\begin{array}{l}\text { Supraorbital } \\
\text { contralateral } \\
\text { to anode } \\
\text { placement }\end{array}$ & $10(5 \mathrm{pw})$ \\
\hline Kaski et a ${ }^{50}$ & 2014 & Gait & & & $\begin{array}{l}\text { Anode: } 40 \\
\text { cathode: } 40\end{array}$ & 2 & Anterior to $\mathrm{Cz}$ & Inion & I \\
\hline $\begin{array}{l}\text { Manenti } \\
\text { et } \mathrm{al}^{54}\end{array}$ & 2014 & Gait & & & 35 & 2 & $\begin{array}{l}\text { Left then right } \\
\text { DLPFC for all } \\
\text { patients }\end{array}$ & $\begin{array}{l}\text { Contralateral } \\
\text { supraorbital }\end{array}$ & 2 \\
\hline $\begin{array}{l}\text { Valentino } \\
\text { et } \mathrm{al}^{56}\end{array}$ & 2014 & Gait & & & Not stated & 2 & $\begin{array}{l}\text { MI corresponding } \\
\text { to leg, patient } \\
\text { started walking } \\
\text { post freezing }\end{array}$ & $\begin{array}{l}\text { Contralateral } \\
\text { orbitofrontal }\end{array}$ & 5 \\
\hline $\begin{array}{l}\text { Biundo } \\
\text { et a }\left.\right|^{53}\end{array}$ & 2015 & & $\mathrm{MCl}$ & & & 2 & Left DLPFC & $\begin{array}{l}\text { Right } \\
\text { supraorbital }\end{array}$ & $16(4 \mathrm{pw})$ \\
\hline \multirow[t]{3}{*}{$\begin{array}{l}\text { Salimpour } \\
\text { et } \mathrm{al}^{31}\end{array}$} & 2015 & UL & & & $\mathrm{N} / \mathrm{A}$ & N/A & $\begin{array}{l}\text { Experiment I: No } \\
\text { stimulation }\end{array}$ & $\begin{array}{l}\text { No } \\
\text { stimulation }\end{array}$ & N/A \\
\hline & & & & & 25 & I & $\begin{array}{l}\text { Experiment 2: } \\
\text { Left } \mathrm{MI}\end{array}$ & Right MI & I \\
\hline & & & & & 25 (assumed) & 2 & $\begin{array}{l}\text { Experiment 3: } \\
\text { Right MI }\end{array}$ & Left MI & I \\
\hline
\end{tabular}




\begin{tabular}{|c|c|c|c|c|c|c|c|c|}
\hline $\begin{array}{l}\text { Duration } \\
\text { per session } \\
\text { (minutes) }\end{array}$ & Study type & Adjunct & $\begin{array}{l}\text { Disease } \\
\text { state } \\
\text { (HY) }\end{array}$ & Sample & Active & Sham & Outcomes & Result \\
\hline 20 & $\begin{array}{l}\text { Single-blind } \\
\text { (assumed); cross- } \\
\text { over }\end{array}$ & & & 9 & 9 & 9 & $\begin{array}{l}\text { Three-back letter working } \\
\text { mem paradigm }\end{array}$ & Nonsignificant \\
\hline 20 & $\begin{array}{l}\text { Single-blind } \\
\text { (assumed); cross- } \\
\text { over }\end{array}$ & & & 9 & 9 & 9 & $\begin{array}{l}\text { Three-back letter working } \\
\text { mem paradigm }\end{array}$ & $\begin{array}{l}\text { Significant (DLPFC } \\
\text { only) }\end{array}$ \\
\hline 20 & $\begin{array}{l}\text { Double-blind; } \\
\text { cross-over }\end{array}$ & & $2.4 \mathrm{HY}$ & 9 & 9 & 9 & MEP; UPDRS; sRT; PPT & Significant \\
\hline 20 & $\begin{array}{l}\text { Double-blind; } \\
\text { cross-over }\end{array}$ & & & 8 & 8 & 8 & MEP; UPDRS; sRT; PPT & Nonsignificant \\
\hline 20 & $\begin{array}{l}\text { Double-blind; } \\
\text { cross-over }\end{array}$ & & & 9 & 9 & 9 & UPDRS; sRT; PPT & Significant \\
\hline 20 & Double-blind & & $2.4 \pm 0.2$ & 25 & 13 & 12 & $\begin{array}{l}10 \text { m walk; UL } \\
\text { bradykinesia; UPDRS; sRT; } \\
\text { BDI; health survey; SelfAx } \\
\text { of mobility }\end{array}$ & Nonsignificant \\
\hline 20 & $\begin{array}{l}\text { Crossover; } \\
\text { no blinding ( } 2 \\
\text { montages tested) }\end{array}$ & & $\begin{array}{l}\mathrm{I} .6 \pm 0.5 \\
\mathrm{HY}\end{array}$ & 16 & 32 & 0 & $\begin{array}{l}\text { Verbal fluency (fixation, } \\
\text { repetition, semantic, } \\
\text { phonemic); fMRI }\end{array}$ & $\begin{array}{l}\text { Significant (DLPFC } \\
\text { over TPC) }\end{array}$ \\
\hline 15 & $\begin{array}{l}\text { Double-blind; } \\
\text { cross-over }\end{array}$ & & $\mathrm{I}-4 \mathrm{HY}$ & 20 & 20 & 20 & $\begin{array}{l}\text { Sit to stand; functional } \\
\text { reach; } 180^{\circ} \text { turn; TUG; } \\
10 \mathrm{~m} \text { walk }\end{array}$ & Nonsignificant \\
\hline 20 & $\begin{array}{l}\text { Double-blind; } \\
2 \text { site }\end{array}$ & & & 18 & II (6+5) & 7 & $\begin{array}{l}\text { TMT A\&B; WCST; PCL; } \\
\text { WM; Stroop; HPVOT; } \\
\text { CPM; MMSE; BDI; HAS; } \\
\text { UPDRS III; sRT; 4-CRT; } \\
\text { PPT; FT; WT; BU; SP }\end{array}$ & $\begin{array}{l}\text { Significant (cog not } \\
\text { motor) }\end{array}$ \\
\hline 15 & $\begin{array}{l}\text { Double-blind; } \\
\text { RCT; cross-over }\end{array}$ & $\begin{array}{l}\text { Physical } \\
\text { therapy during } \\
\text { stimulation }\end{array}$ & & 16 & 8 & 8 & Gait velocity; TUG & Significant \\
\hline 7 & $\begin{array}{l}\text { Double-blind; } 2 \\
\text { montages tested } \\
\text { on each patient } \\
\text { L\&R DLPFC }\end{array}$ & & $1.3 \pm 1.1$ & 10 & 20 & 10 & TUG & $\begin{array}{l}\text { Significant (right } \\
\text { DLPFC) }\end{array}$ \\
\hline 20 & $\begin{array}{l}\text { Double-blind; } \\
\text { cross-over }\end{array}$ & & 2-4 HY & 10 & 10 & 10 & $\begin{array}{l}\text { Motor UPDRS; stand- } \\
\text { walk-sit }\end{array}$ & Significant \\
\hline 20 & Double-blind & $\begin{array}{l}\text { Computer- } \\
\text { based cog } \\
\text { training }\end{array}$ & & 24 & 12 & 12 & RBANS & Significant \\
\hline N/A & $\mathrm{N} / \mathrm{A}$ & & $\mathrm{I}-2.5 \mathrm{HY}$ & $\begin{array}{l}15 \text { PD + } \\
15 \text { Healthy }\end{array}$ & $N / A$ & $\mathrm{~N} / \mathrm{A}$ & $\mathrm{N} / \mathrm{A}$ & $N / A$ \\
\hline 25 & Not stated & & & 10 & 10 & 0 & $\begin{array}{l}\text { UPDRS (motor assumed); } \\
\text { bimanual UL task; } \\
\text { unimanual task; unimanual } \\
\text { max force }\end{array}$ & Nonsignificant \\
\hline 25 & Not stated & & & 10 & 10 & 0 & $\begin{array}{l}\text { Motor UPDRS; bimanual } \\
\text { UL task; unimanual task; } \\
\text { unimanual max force }\end{array}$ & Significant \\
\hline
\end{tabular}


Table I (Continued)

\begin{tabular}{|c|c|c|c|c|c|c|c|c|c|}
\hline Authors & Year & Motor & Cognitive & Other & $\begin{array}{l}\text { Electrode } \\
\text { size }\left(\mathrm{cm}^{2}\right)\end{array}$ & $\begin{array}{l}\text { Electrode } \\
\text { intensity } \\
(\mathrm{mA})\end{array}$ & Anode & Cathode & Sessions \\
\hline & & & & & 25 (assumed) & Not stated & $\begin{array}{l}\text { Experiment 4: } \\
\text { Right or left MI }\end{array}$ & $\begin{array}{l}\text { Right or left } \\
\text { MI }\end{array}$ & $\begin{array}{l}3 \\
\text { (consecutive } \\
\text { days) }\end{array}$ \\
\hline & & & & & 25 (assumed) & 2 & $\begin{array}{l}\text { Experiment 5: } \\
\text { Ipsilateral to } \\
\text { affected side }\end{array}$ & $\begin{array}{l}\text { Contralateral } \\
\text { to affected } \\
\text { side }\end{array}$ & $\begin{array}{l}5 \text { (day I: } \\
\text { measures; } \\
\text { days 2-3: } \\
\text { sham; days } \\
\text { 4-8: stim; } \\
\text { days 9-10: } \\
\text { measures) }\end{array}$ \\
\hline $\begin{array}{l}\text { Costa- } \\
\text { Ribeiro } \\
\text { et al }{ }^{49}\end{array}$ & 2017 & Gait & & & 35 & 2 & $\begin{array}{l}2 \mathrm{~cm} \text { anterior to } \\
\text { vertex in sagittal } \\
\text { midline }\end{array}$ & $\begin{array}{l}\text { Supraorbital } \\
\text { area of most } \\
\text { affected side }\end{array}$ & $10(2 / 3 \mathrm{pw})$ \\
\hline $\begin{array}{l}\text { Costa- } \\
\text { Ribeiro } \\
\text { et a }\left.\right|^{49}\end{array}$ & 2016 & Gait & & & 35 & 2 & $\mathrm{Cz}$ & $\begin{array}{l}\text { Supraorbital } \\
\text { contralateral } \\
\text { to most } \\
\text { affected side }\end{array}$ & $10(3 \mathrm{pw})$ \\
\hline \multirow[t]{2}{*}{$\begin{array}{l}\text { Ferrucci } \\
\text { et } \mathrm{al}^{33}\end{array}$} & 2016 & Dyskinesia & & & 35 & 2 & $\begin{array}{l}\mathrm{I}-2 \mathrm{~cm} \text { below } \\
\text { inion }\end{array}$ & Shoulder & $5+$ sham \\
\hline & & & & & 35 & 2 & Left and right $\mathrm{MI}$ & Right deltoid & $5+$ sham \\
\hline $\begin{array}{l}\text { Manenti } \\
\text { et } \mathrm{a}^{52}\end{array}$ & 2016 & & $\mathrm{MCl}$ & & 35 & 2 & $\begin{array}{l}\text { DLPFC - } \\
\text { contralateral to } \\
\text { most affected side }\end{array}$ & $\begin{array}{l}\text { Ipsilateral } \\
\text { supraorbital }\end{array}$ & $10(5 \mathrm{pw})$ \\
\hline $\begin{array}{l}\text { Schabrun } \\
\text { et } \mathrm{al}^{30}\end{array}$ & 2016 & Gait & & & 35 & 2 & Left MI & $\begin{array}{l}\text { Contralateral } \\
\text { supraorbital }\end{array}$ & $9(3 \mathrm{pw})$ \\
\hline Swank et a ${ }^{55}$ & 2016 & Gait & & & Not stated & 2 & Left DLPFC & Right DLPFC & $\begin{array}{l}\text { I active; I } \\
\text { sham }\end{array}$ \\
\hline $\begin{array}{l}\text { Forogh } \\
\text { et } \mathrm{al}^{82}\end{array}$ & 2017 & & & $\begin{array}{l}\text { Fatigue/ } \\
\text { daytime } \\
\text { sleepiness }\end{array}$ & 35 & 2 (assumed) & Left DLPFC & Right DLPFC & 8 (2 weeks) \\
\hline Lattari et $\mathrm{a}^{51}$ & 2017 & $\begin{array}{l}\text { Balance and } \\
\text { gait }\end{array}$ & & & 35 & 2 & Left DLPFC & $\begin{array}{l}\text { Right } \\
\text { supraorbital } \\
\text { cortex }\end{array}$ & I \\
\hline
\end{tabular}

Abbreviations: DLPFC, dorsolateral prefrontal cortex; Exec, executive function; MI, primary motor cortex; MCl, mild cognitive impairment; PD, Parkinson's disease; TPC, temporoparietal cortex; UL, upper limb; sRT, simple reaction time; UPDRS, Unified Parkinson's Disease Rating Scale; MEP, motor-evoked potential; tDCS, transcranial direct current stimulation; fMRI, functional MRI. N/A, not available; HY, Hoehn \& Yahr; RCT, randomized controlled trial; PPT, Purdue Pegboard Test; WCST, Wisconsin Card Sorting Test; CPM, Colored Progressive Matrices; MMSE Mini mental state examination; HPVOT, Hooper Visual Organization Test; HAS, Hamilton Anxiety Scale; 4-CRT, 4-choice reaction time; FT, Finger Tapping; WT, walking time; BU, buttoning up; SP supination-pronation; RBANS, Repeatable battery assessment of neuropsychological status; aMT, active motor threshold; PDQ8 Parkinson's disease quality of life questionnaire; VAT, visual attention task; PDCRS, parkinson's disease cognitive rating scale; FSI, fatigue severity index; ESS, Epworth Sleepiness Scale; DGI, dynamic gait index; Berg, Berg Balance Scale; UL-MT, upper limb motor task; stim, stimulation; SelfAx, selfassessment; BDI, Beck Depression Index; TUG, timed up and go; cog, cognition; L\&R, left and right; PCL, probabilistic classification learning; TMT A\&B, trial making tests A\&B; WM, working memory; pw, per week; TPC, temporoparietal cortex; Cz, central electrode in I0-20 electroencephalogram placement.

the time taken to complete a variety of motor sequences 10 times as a measure of bradykinesia. They found no benefit of tDCS above and beyond the gait training.

Salimpour et $\mathrm{al}^{31}$ conducted a study to evaluate the subjective cost of producing force with either upper limb in patients with PD. They applied simultaneous tDCS to both the motor cortices to alter the degree of "noise" associated with a particular limb movement. As a part of this study, they performed a double-blind assessment in 10 PD patients and performed a clinical examination on 3 separate days. On each day, subjects received anodal, cathodal, or sham stimulation over the motor cortex contralateral to the affected side. The order of stimulation was randomized and blinded to the clinical raters. They observed a significant improvement in UPDRS following cathodal stimulation compared to both anodal stimulation and sham. UPDRS showed only a trend 


\begin{tabular}{|c|c|c|c|c|c|c|c|c|}
\hline $\begin{array}{l}\text { Duration } \\
\text { per session } \\
\text { (minutes) } \\
\end{array}$ & Study type & Adjunct & $\begin{array}{l}\text { Disease } \\
\text { state } \\
\text { (HY) }\end{array}$ & Sample & Active & Sham & Outcomes & Result \\
\hline Not stated & $\begin{array}{l}\text { Double-blind; } 3 \\
\text { montages tested } \\
\text { (contra MI } \\
\text { anode; contra MI } \\
\text { cathode; sham) }\end{array}$ & & & 10 & 10 & 0 & $\begin{array}{l}\text { Motor UPDRS; (bimanual } \\
\text { UL task; unimanual task; } \\
\text { unimanual max force - all } \\
\text { assumed) }\end{array}$ & $\begin{array}{l}\text { Significant (cathodal } \\
\text { montage only) }\end{array}$ \\
\hline Not stated & $\begin{array}{l}\text { Not stated - } \\
\text { single-blind } \\
\text { (assumed) }\end{array}$ & & & 8 & 8 & 8 & $\begin{array}{l}\text { Motor UPDRS; bimanual } \\
\text { UL task; unimanual task; } \\
\text { unimanual max force }\end{array}$ & Significant \\
\hline 13 & Double-blind & Gait training & $\mathrm{I}-3 \mathrm{HY}$ & 24 & 11 & II & TUG; UL-MT; MEP; aMT & Significant \\
\hline 13 & $\begin{array}{l}\text { Double-blind; } \\
\text { RCT }\end{array}$ & $\begin{array}{l}\text { Cued gait } \\
\text { training }\end{array}$ & & 24 & 11 & II & $\begin{array}{l}10 \text { m walk; TUG; cadence; } \\
\text { stride }\end{array}$ & Nonsignificant \\
\hline 20 & $\begin{array}{l}\text { Double-blind; } \\
\text { cross-over }\end{array}$ & & 2-3 HY & 9 & 9 & 4 & $\begin{array}{l}\text { UPDRS III \&IV; PDQ-8; } \\
\text { BDI; VAT; sRT }\end{array}$ & Significant \\
\hline 20 & $\begin{array}{l}\text { Double-blind; } \\
\text { cross-over }\end{array}$ & & 2-3 HY & 9 & 9 & 5 & $\begin{array}{l}\text { UPDRS III \&IV; PDQ-8; } \\
\text { BDI; VAT; sRT }\end{array}$ & Significant \\
\hline 25 & Double blind & $\begin{array}{l}\text { Physical } \\
\text { therapy during } \\
\text { stimulation }\end{array}$ & $\begin{array}{l}2.2 / 2.3 \\
\mathrm{HY}\end{array}$ & 20 & 10 & 10 & PDCRS & Significant \\
\hline 20 & $\begin{array}{l}\text { Double-blind; } \\
\text { parallel group } \\
\text { design }\end{array}$ & Gait training & 2-3 HY & 16 & 8 & 8 & $\begin{array}{l}\text { Gait speed, step } \\
\text { length, cadence, TUG, } \\
\text { bradykinesia, motor speed }\end{array}$ & Nonsignificant \\
\hline 20 & $\begin{array}{l}\text { Single-blind } \\
\text { (assumed); cross- } \\
\text { over }\end{array}$ & & $\begin{array}{l}2 \mathrm{HY} \\
\text { (median) }\end{array}$ & 10 & 10 & 10 & $\begin{array}{l}\text { TUG (alone); TUG } \\
\text { (manual); TUG (cognitive); } \\
\text { PDQ-39 }\end{array}$ & Nonsignificant \\
\hline 20 & Double-blind & $\begin{array}{l}\text { Occupational } \\
\text { therapy }\end{array}$ & & 23 & 12 & 11 & FSI; ESS & $\begin{array}{l}\text { Significant (fatigue } \\
\text { only) }\end{array}$ \\
\hline 20 & $\begin{array}{l}\text { Double-blind; } \\
\text { cross-over }\end{array}$ & & $2.35 \pm 1.06$ & 17 & 17 & 17 & Berg; DGI; TUG & $\begin{array}{l}\text { Significant - all } \\
\text { measures }\end{array}$ \\
\hline
\end{tabular}

toward significant change after anodal stimulation $(p=0.06)$. These results are contrary to those of Benninger et $\mathrm{al}^{26}$ who also used M1 stimulation, although they did not specifically stimulate contralateral to the affected side. Moreover, Salimpour et al ${ }^{31}$ did not test patients in the "off" medication condition, which was associated with the greatest clinical improvement in motor function in the Benninger study.

\section{tDCS for tremor in PD}

There have been very few studies of tDCS focusing specifically on the treatment of tremor in PD. In their randomized, double-blind, sham-controlled study design, Benninger et $\mathrm{al}^{26}$ found no improvement in self-reported tremor scores using anodal tDCS over the premotor and motor or prefrontal cortices. In addition, although the study was not powered to perform comparative analysis of UPDRS subscores, Fregni et $\mathrm{a}^{25}$ reported a $10 \%$ improvement in tremor scores on the UPDRS with anodal tDCS over M1.

\section{tDCS for dyskinesia in PD}

The development of Levodopa-induced dyskinesia may have a significant negative impact on quality of life among some PD patients and is thought to arise due to aberrant plasticity within the denervated striatum. ${ }^{32}$ Recently, Ferrucci et $\mathrm{al}^{33}$ 
investigated the effect of tDCS on dyskinesia, motor, and cognitive domains. In this sham-controlled study, nine participants received two active stimulation protocols in a random order. Motor cortex stimulation consisted of anodal stimulation to left and right M1 (with a third electrode - the cathode - placed over the deltoid). Cerebellar stimulation utilized a previously validated protocol, with the anode placed 1-2 $\mathrm{cm}$ below the inion and the reference electrode positioned over the shoulder. Active stimulation was delivered over 5 consecutive days for 20 minutes at $2 \mathrm{~mA}$ intensity. Compared to baseline, dyskinesias (measured using the UPDRS IV - Dyskinesia) on the final day of stimulation (day 5) showed a significant improvement from baseline in both the active experiments and compared to sham stimulation. Interestingly, the authors saw no change in other motor or cognitive domains.

\section{tDCS for gait in PD}

Postural instability and gait dysfunction are cardinal motor features of PD, often leading to significant reduction in quality of life $^{34}$ and predispose sufferers to falls, with associated increased morbidity and mortality. ${ }^{35,36}$ Parkinsonian gait is characterized by reduced step and stride length, shuffling of the feet, difficulty turning, and freezing of gait (FOG). An appreciation of the neuronal mechanisms underpinning gait and postural disturbance in PD can help guide therapeutic treatments using tDCS in relation to the most appropriate electrode montages to use. In healthy individuals, the supplementary motor area (SMA) displays high activity during internally driven movements, while premotor areas are relatively more involved when a movement is externally cued. ${ }^{37}$ However, in patients with $\mathrm{PD}$, the activity within the SMA is reduced, which may be related to the difficulty in preparing instructions to trigger a movement. ${ }^{38,39}$

Premotor and primary motor regions appear to be relevant therapeutic targets for patients with PD and gait disturbance. ${ }^{40}$ On the basis of this, Benninger et $\mathrm{a}^{26}$ studied the change in the timed test of gait in the "on" and "off" state 24 hours after the tDCS intervention period compared with baseline. Assessments were taken over a 3-month period. Twenty-five PD patients were investigated, 13 receiving tDCS and 12 sham stimulation. tDCS led to a significant decrease in walking time with the removal of one outlier from the sham stimulation group ( $-22.6 \%$ vs $3.6 \% ; p=0.002)$. No differences were seen when "on" $(-17.4 \%$ vs $-12.7 \% ; p=0.44)$ or beyond the immediate post-intervention period at 1 or at 3 months thereafter.

Verheyden et $\mathrm{al}^{41}$ conducted a double-blind, experimental crossover study with 20 PD patients undergoing either active
(1 $\mathrm{mA}$ for 15 minutes) or sham tDCS. The anode was placed over M1 of the dominant hemisphere and the cathode over the contralateral supraorbital region. Each patient acted as their own control and was assessed in the "on" medication state. Patients performed a battery of clinical measures prior to tDCS, namely timed sit-to-stand, functional reach, standing-start $180^{\circ}$ turning, Timed Up and Go (TUG) test, and $10 \mathrm{~m}$ walk test. Either active or sham tDCS was then delivered while the participants repeated the measures. Finally, the outcome measures were collected again following the stimulation. The authors observed no significant improvement with active tDCS across any outcome. In fact, there was a decline in the time taken to complete the $10 \mathrm{~m}$ walk following active tDCS. Given that PD may show hemispheric lateralization, one explanation offered for the negative result was that application over a single hemisphere may in fact alter inter-hemispheric balance and lead to relatively impaired functional outcomes, that is, increasing cortical excitability of one hemisphere may lead to relative decrease in cortical excitability of the other hemisphere through interhemispheric inhibition. ${ }^{42}$

\section{Combining tDCS with cueing and/ or physical training}

It was hypothesized that the combination of tDCS with physical therapies could optimize the neuroplastic changes induced by motor practices and lead to greater and longer-lasting clinical gains in neurological rehabilitation. Cueing is an established technique that utilizes somatosensory, auditory, and visual information to provide timing or spatial stimuli to facilitate the initiation and continuation of motor activity. Several clinical trials indicate cueing therapy-induced improvements in walking speed, step length, and frequency in PD. ${ }^{43-46}$ From a neuroanatomical perspective, enhanced activation of the premotor cortex seems to be relevant for the improvement of motor performance induced by cueing therapy. ${ }^{47}$

Thus, Costa-Ribeiro et al ${ }^{29}$ investigated the effects of tDCS combined with cued gait training (CGT) on functional mobility in patients with PD. They conducted a pilot doubleblind controlled, randomized clinical trial with patients assigned either to anodal tDCS with CGT $(n=12$, one lost to follow-up) or to sham tDCS with CGT ( $n=12$, one lost to follow-up). Functional mobility was assessed by $10 \mathrm{~m}$ walk test, cadence, stride length, and TUG. All the outcomes improved with CGT except stride length and TUG test performance. However, gait training effects associated with visual cues were not influenced by tDCS on any of these outcomes. 
Interestingly, TUG and gait velocity showed improvement gains to be maintained at 1 month only in the active tDCS group. These results suggest that $\mathrm{tDCS}$ may indeed prolong the effectiveness of cued therapies in PD patients, but in itself it does not increase the effect size achieved with CGT alone. Different stimulation areas between studies are a potential factor to explain the differences observed in the results, given that stimulation over premotor and primary motor areas seem to be more effective in improving gait in PD compared to SMA stimulation. Indeed, a separate study using an externally guided task also failed to show the after-effects of repetitive TMS over the SMA on performance of a tapping task. ${ }^{48}$

In a follow-up randomized, double-blind, controlled study, ${ }^{49}$ the same authors investigated the dopaminedependent effect of combining tDCS with visual CGT. They evaluated the TUG as a measure of functional mobility in 22 patients with PD. The experimental group $(n=11)$ received active anodal tDCS over the SMA and the control group $(n=11)$ sham tDCS, in both "on" and "off" medication states. Both the groups received visual CGT following the tDCS. Functional mobility improved in either "on" or "off" medication conditions compared with baseline, irrespective of tDCS. However, for both the medication conditions, these improvements were maintained at 1 month only in the experimental group. These findings further support that tDCS can prolong the positive effect induced by CGT on functional mobility but that such effects are unrelated to dopaminergic medication.

One possible explanation for the lack of efficacy of tDCS upon gait observed in these studies is that given the bilateral representation of cortical gait regions, one might expect improvements in gait to require simultaneous modulation of cortical activity bilaterally. Although cortical leg stimulation using non-invasive techniques is more challenging than upper limb cortical stimulation given its deeper anatomical location, and more vertical orientation than the hand motor cortex, experimental evidence has shown that a single anode electrode placed over right and left M1 and premotor cortices can alter cortical excitability in both the legs simultaneously. On the basis of this, Kaski et $\mathrm{al}^{50}$ combined a midline anodal tDCS montage (15 minutes of $2 \mathrm{~mA}$ current over midline primary motor and premotor cortex) with physical training in a double-blind, cross-over design. One group $(n=8)$ underwent gait and balance training during tDCS. Patients received the opposite stimulation with physical training 1 week later. The second group $(n=8)$ received stimulation but no training, and also repeated a tDCS session 1 week later. Physical training consisted of a systematic set of exercises relating to gait and balance repeated over a single 30-minute session. Outcomes included gait velocity, stride length, timed 6-minute walk test, TUG, and performance on the pull test. The combination of tDCS with physical training increased gait velocity ( mean $=29.5 \%, \mathrm{SD}=13 ; p<0.01$ ) and improved balance above and beyond the effects of tDCS alone. Greater tDCS-related improvements were observed in patients with higher UPDRS scores (ie, more severe disease).

Schabrun et al did not find any benefit of tDCS above and beyond physical training. ${ }^{30}$ They investigated the feasibility and safety of combining anodal tDCS with a dual task gait training intervention in 16 patients with PD. They performed a pilot, randomized, double-blind, sham-controlled parallel group trial with 12-week follow-up. Patients received nine dual task gait training sessions over 3 weeks and were randomized to receive active or sham tDCS over the first 20 minutes of each session. Outcomes included gait speed, step length, cadence, and TUG, all during a concurrent cognitive task (eg, counting). Gait speed, step length, and cadence improved in both the groups, under all dual task conditions. This effect was maintained at follow-up. There was no difference between the active and sham tDCS groups.

While most studies of tDCS assessing gait and balance in PD have focused on the primary motor and premotor cortex, Lattari et $\mathrm{al}^{51}$ investigated the effects of active (anodal) stimulation over the left DLPFC ( $2 \mathrm{~mA}$ for 20 minutes) on balance and mobility in 17 patients with $\mathrm{PD}$, on medication. They ran a double-blind, randomized, cross-over trial with each participant undergoing anodal tDCS and sham tDCS, with a minimum washout period of 48 hours. They recorded the Berg Balance Scale, Dynamic Gait Index and TUG. DLPFC anodal tDCS improved all measures as compared to sham stimulation. The authors argue that reduced SMA activity in patients with PD leads to enhanced activity in other cortical regions, including the DLPFC, thus accounting for motor improvements in their study. Alternatively, tDCS may have enhanced visuo-spatial processing, or indeed activated neighboring lateral prefrontal regions involved in compensating for reduced SMA activity.

Manenti et $\mathrm{al}^{52}$ also investigated repeated sessions of DLPFC tDCS combined with physical training upon motor and cognitive performance. The authors administered $2 \mathrm{~mA}$ anodal tDCS to the DLPFC contralateral to the patients' most affected side, together with physical therapy for 25 minutes a day, 5 days a week, for 2 weeks. They found no significant benefit of tDCS compared to sham stimulation in tests of gait and stance, although both the groups improved over time, likely as a result of both learning effects and physical therapy; there was however a reported improvement in 
cognitive performance. Similar findings on motor function (UPDRS part III) were found by Biundo et $\mathrm{al}^{53}$ using a similar stimulation and therapy protocol. While this study did not focus on motor outcomes, no significant shift in motor scores following repeated sessions of stimulation was observed; however, a trend for improved performance in specific cognitive domains was reported.

In an earlier study, Manenti et $\mathrm{al}^{54}$ reported improved gait performance, as measured by the TUG immediately following anodal tDCS of the right DLPFC. They rationalized that frontal areas are active during locomotion and that tDCS may play a role in the modulation of prefrontal dopamine release or work to stimulate frontal regions responsible for executive function and hence gait. The study involved 10 subjects who were administered a modest dose of $2 \mathrm{~mA}$ anodal tDCS for 7 minutes to the left and right DLPFC (on different days) and who also received sham stimulation. TUG scores were recorded at baseline and following stimulation. Interestingly, eight of the subjects were reported to have left side of the body as more affected by PD.

Swank et $\mathrm{al}^{55}$ stimulated the left DLPFC with anodal tDCS and compared this to sham stimulation, measuring subject performance completing the TUG under three different conditions: 1) TUG alone; 2) TUG while holding a cup of water; 3) TUG while counting backwards. Active stimulation was delivered for 20 minutes at $2 \mathrm{~mA}$ to 10 subjects. The authors did not report whether any participants exhibited lateralized symptoms or presented with a more affected side of the body. Interestingly, the active stimulation protocol appeared to have no meaningful effect on TUG alone, however did influence the performance on TUG while holding a cup of water, and most considerably on the TUG while counting backwards. This is in keeping with the role of the DLPFC in cognition.

FOG is considered a discreet clinical entity but may also be a feature of PD that represents a disabling motor complication. Valentino et $\mathrm{al}^{56}$ were the first to investigate the efficacy of tDCS of the primary motor cortex of PD patients with FOG. In this double-blind, sham-controlled, cross-over study, 10 PD patients with FOG in the "on" medication state underwent 20 minutes of anodal ( $2 \mathrm{~mA}$ ) or sham stimulation across 5 consecutive days. Clinical assessments were performed over a month. Active stimulation led to a significant improvement in the Stand Walk Sit test and a reduction in the frequency and duration of FOG episodes. The authors also observed an improvement in the motor (part III) and total UPDRS with active compared to sham stimulation. Beneficial effects were more evident after the entire 5-day stimulation session and persisted until the end of the observation period.
The authors suggest that anodal tDCS may have stimulated the release of dopamine through activation of glutamatergic cortico-striatal projections. ${ }^{57,58}$ Alternatively, or additionally, tDCS may have induced an excitatory modulation of the cortico-striato-thalamo-cortical circuits that have been shown to be impaired in patients with FOG. ${ }^{59}$

\section{tDCS for cognition}

Cognitive functions are predominantly executed by the cortex, where dopamine is known to be a key neurotransmitter. ${ }^{60}$ It has been suggested that disruption of the dopaminergic system, as occurs in PD, may be one mechanism involved in cognitive impairment. ${ }^{61}$ Indeed, cognitive impairment is a common feature of PD, and although it tends to occur as a later manifestation of the condition, it is an important predictor of quality of life in these patients. The characteristics of the cognitive impairment observed in PD patients include deficits of executive function, such as planning and working memory. ${ }^{62,63}$ Depression in PD has also been associated with changes in dopaminergic transmission across cortical and subcortical areas, and aberrant neuronal excitability between the right and left DLPFC may underpin some of the psychiatric manifestations in patients with $\mathrm{PD} .{ }^{64}$ Indeed, anodal tDCS over the left DLPFC with the cathode over the right supraorbital region has been shown to improve working memory in healthy subjects, ${ }^{65}$ as well as improve mood in patients with major depression. ${ }^{66,67}$ In this context, several studies have documented the beneficial effects of TMS on cognitive symptoms in $\mathrm{PD}^{68,69}$ without worsening motor symptoms. ${ }^{70}$ These results support the idea that active stimulation of the DLPFC with tDCS could also have beneficial effects on both affective and cognitive domains in patients with PD.

Boggio et $\mathrm{al}^{71}$ investigated the differential effects of $1 \mathrm{~mA}$ versus $2 \mathrm{~mA}$ anodal tDCS over the left DLPFC, M1 or sham on the performance of a working memory task in 18 PD patients. A significant improvement in working memory accuracy, but not speed, was observed only after left DLPFC tDCS at $2 \mathrm{~mA}$. The results of this study are in line with neuroimaging and rTMS evidence showing that the left DLPFC is critical to working memory formation. ${ }^{72-73}$ Surprisingly, stimulation at $1 \mathrm{~mA}$ did not induce significant effects upon working memory in these patients. The authors hypothesized that tDCS may be less effective in older subjects and thus higher currents would be required. In support of this hypothesis, lesser effects of non-invasive brain stimulation have been observed in the elderly compared to a younger population. ${ }^{74}$ Nevertheless, other studies ${ }^{25}$ have shown that $1 \mathrm{~mA}$ stimulation for 20 minutes is able to induce behavioral 
effects in PD patients of a similar age to those included in the Boggio et $\mathrm{al}^{71}$ study. A further possibility is that a $1 \mathrm{~mA}$ stimulation would be insufficient to induce a significant release of dopamine, a possibility supported by studies in healthy subjects. ${ }^{75}$

Doruk et $\mathrm{al}^{28}$ conducted a multicenter, sham-controlled, double-blind study to assess the immediate and long-term effects of 10 consecutive sessions of tDCS. Anodal stimulation was delivered either over the right DLPFC $(n=5)$, left DLPFC $(n=6)$, or sham $(n=7)$. Cognitive function, depressive symptoms, and motor functions were evaluated in 18 PD patients at baseline, at the end of the 2-week stimulation sessions, and at 1-month follow-up. Their results were mixed, showing an improvement in a test of executive function (trail making test B) across all groups (including sham, thus likely to represent a learning effect), but improvements were only sustained at 1-month follow-up in the active left and right DLPFC stimulation groups. Such effects were not seen for a variety of other cognitive tasks. While the results suggest the existence of a beneficial long-term effect on executive function in PD patients following active tDCS over the DLPFC, the authors found no additional benefit of left versus right DLPFC stimulation. The authors also reported improvement in depressive symptoms at the end of the 10-session intervention, only for left DLPFC tDCS, although baseline depression scores were remarkably low in this study. Benninger et $\mathrm{al}^{26}$ found no significant improvement in depression scores on the Beck Depression Inventory following tDCS to the motor and prefrontal cortex, but again baseline values were low.

Pereira et $\mathrm{al}^{76}$ compared the effects induced by frontal versus temporoparietal tDCS upon phonemic and semantic fluency functional networks in patients with PD. They randomized 16 patients with PD to receive tDCS ( $2 \mathrm{~mA}$ for 20 minutes) to either the left DLPFC or left temporoparietal cortex (TPC) in a counterbalanced order. Immediately following stimulation, patients underwent a verbal fluency paradigm inside a functional MRI scanner. They assessed differences in functional connectivity associated with the two different stimulation protocols. In addition, and in light of abnormalities in default-mode networks observed in $\mathrm{PD},{ }^{77,78}$ the authors also evaluated the effects of tDCS on task-related pattern networks (a distributed brain system normally deactivated during attention-demanding tasks) that have neuroanatomical overlap with default-mode networks. DLPFC tDCS led to greater enhancement of functional connectivity in verbal fluency and greater deactivation in task-related networks than stimulation over TPC. In addition, DLPFC tDCS increased performance on the phonemic fluency task hinting at a relationship between neurobiologic and behavioral effects. Although the authors argue that $\mathrm{tDCS}$ stimulation effects on brain activity are limited to 10 minutes ${ }^{79}$ or 15 minutes, ${ }^{80}$ their washout period of only 2 hours may have been a significant confounder. Moreover, the authors did not include a sham stimulation protocol which makes it difficult to exclude an order effect on performance.

\section{tDCS with cognitive training}

Recently, Manenti et al ${ }^{52}$ demonstrated stimulation to the DLPFC contralateral to the patients' most symptomatic side was effective at improving cognitive performance among PD subjects compared to sham. In this repeated session experiment, there was improvement immediately following, and persistent at 3 months after, stimulation. An overall improvement to motor and depressive symptoms was observed in both the groups (active and sham) and attributed to physical therapy which was provided as an adjunct to all participants.

Manenti et $\mathrm{al}^{52}$ compared the short- and long-term effects of active versus sham tDCS combined with physical therapy in PD with mild cognitive impairment. PD patients with mild cognitive impairment present subtle cognitive dysfunctions, such as memory deficits and difficulties in frontal/executive abilities, with preserved activities of daily living. ${ }^{81}$ This condition is associated with a higher risk of developing Parkinson's dementia. The authors administered $2 \mathrm{~mA}$ tDCS DLPFC together with physical therapy for 25 minutes a day, 5 days a week, for 2 weeks. They found significant improvements in the PD cognitive rating scale, frontal subcortical scores, and verbal fluency task, with a stable effect even at 3 months. A similar trend toward significance was observed for the trail making test B (a test of executive function). As expected, there were no improvements in a visual memory task (not reliant upon the DLPFC) with tDCS, excluding a general, nonspecific effect upon cognitive processing. In this repeated session experiment, there was improvement immediately following, and persistent at 3 months after, stimulation. An overall improvement to motor and depressive symptoms was observed in both the groups (active and sham) and attributed to physical therapy which was provided as an adjunct to all participants.

Biundo et al ${ }^{53}$ tested 24 subjects with PD, with 16 stimulation sessions over 4 weeks. The anode was placed over the left DLPFC of subjects and current was administered for $20 \mathrm{~min}$ at $2 \mathrm{~mA}$. Interestingly, the researchers chose to stimulate in conjunction with simultaneous computer-based cognitive training for 30 minutes. They observed a trend for 
improvement in memory skills in the active tDCS group immediately after stimulation and at 4 weeks follow-up (which diminished over time), while another memory-based task showed a delayed benefit only at follow-up, 16 weeks after stimulation, but no other significant effects across a range of cognitive tasks. The authors argue that their use of more stringent criteria to define mild cognitive impairment (MCI) status compared to Manenti et al ${ }^{52}$ may explain the lack of more widespread effects on cognition in their study.

\section{tDCS for fatigue and day-time sleepiness}

Fatigue and sleep abnormalities are common non-motor symptoms of PD. Using two validated questionnaires, Forogh et $\mathrm{al}^{82}$ investigated the effects of anodal tDCS applied over the left DLPFC on fatigue and daytime sleepiness. In a double-blind, sham-controlled design, 23 participants received either sham $(n=11)$ or real $(n=12)$ stimulation for eight sessions (20 min each) over a 2 -week period, with a current equivalent to $2 \mathrm{~mA}$. Both real and sham stimulations were provided in addition to occupational therapy input. At 3 months following stimulation, there was a significant improvement in the real stimulation group for fatigue but not for daytime sleepiness, with no notable change in the sham stimulation group. Despite complex multifactorial causes for sleep disturbances and fatigue, the authors hypothesized that the positive results observed in this study may relate to the established effect of tDCS on mood and depressive symptoms. ${ }^{83}$

\section{Clinical implications}

Of the 19 studies included in this review, 14 showed positive effects (in at least one experimental condition) of tDCS in PD. Thus, taken together, there seems to be strong evidence to support the use of tDCS, either alone or in combination with physical and cognitive therapies in patients with PD and across a range of motor and non-motor symptoms. Why then, has tDCS not yet entered mainstream clinical practice? Two critical reasons are the large variability in study protocols and the small sample sizes studied. Despite significant results across some motor and cognitive domains, differences in outcomes between studies are often attributed to differences in study protocols (eg, delivering $1 \mathrm{~mA}$ versus $2 \mathrm{~mA}$, or stimulating for 15 minutes versus 20 minutes). The presence of multiple pilot studies each using different stimulation protocols hinders progress in designing larger scale studies. Small sample sizes means that it is difficult to draw any firm conclusions, with almost every study reviewed here concluding that there is insufficient power to establish conclusive results. One further limiting factor is the publication bias that exists in the scientific community, with a tendency to publish mostly positive results.

Specific scientific data aside, it is well recognized by technological industries that "implementing new methods, guidelines or tools into routine care is a slow and unpredictable process, and the factors that play a role in the change process are not yet fully understood." ${ }^{44}$ Some of the factors relate to the nature of the innovation itself (eg, the complexity and compatibility of the technology), the characteristics of the adopting organization (eg, attitudes and perceptions), and behavior pattern characteristics of the adopting organization (eg, the size and structure of available resources). This said, tDCS has been around for over 100 years now. Therefore, it is time to encourage large-scale, multicenter, randomized, double-blind, Phase III studies using standardized protocols based on the more robust published pilot data.

\section{Key considerations for a large-scale, multicenter clinical trial of tDCS in PD \\ Effects of $t D C S$ on dopamine}

In a study of anesthetized rats, a 10 minute exposure to cathodal tDCS of the frontal lobe resulted in a $60 \%$ increase in dopamine in the striatum that was sustained for hours after cessation of stimulation. Conversely, anodal stimulation decreased dopamine concentrations by $10 \% .{ }^{20}$ In healthy individuals, anodal tDCS over M1 brings neurons closer to depolarization threshold. ${ }^{85}$ Consistent with animal data, in healthy individuals, the excitatory effects of anodal tDCS upon neuronal excitability are blunted by L-dopa. ${ }^{27}$ This may explain why some studies have not observed positive effects of anodal tDCS in medicated PD patients, ${ }^{26,41}$ though this would be in contrast to other study outcomes. ${ }^{56}$ While "off" medication states may be of research interest, medication withdrawal may be of lesser practical interest in the clinical setting. Nevertheless, pilot data suggest that applying tDCS during medication troughs may increase the potentiation of tDCS effects.

\section{Online versus offline, with or without training?}

The available data suggest that greater effects of tDCS upon motor and cognitive function are achieved through online tDCS application - that is, delivering tDCS during the concurrent performance of a motor/cognitive task. In addition, 
the application of online tDCS seems to be enhanced by concurrent cognitive/motor training. Future interventions need to compare standard (not individualized) and tailored (individualized) cognitive training and examine whether combining cognitive training with brain stimulation further improves cognition in PD.

\section{Lateralization effects}

A clinical feature of PD is the asymmetric onset of motor symptoms, corresponding to contralateral neuronal nigrostriatal degeneration. Indeed, the side of onset may have prognostic implications. ${ }^{86}$ Moreover, cognitive decline in PD has been associated with left-sided onset of motor features, ${ }^{87}$ hinting at an association between laterality of symptom onset and disease progression and severity. Further work is required to better characterize the role of lateralization in PD and possible associated effects of handedness upon disease onset and progression. As such, future studies of tDCS in PD may need to consider the effect of lateralization upon symptom type and disease progression and severity.

\section{Stimulation duration, current density and electrode montages}

At present, studies that have focused on the effect of tDCS on PD lack the sample sizes and statistical power to be interpreted as clinically meaningful to the general population of patients, and in addition, many studies have produced results at variance. The incongruence of many study outcomes can be explained by the vagaries and heterogeneity in study design, including electrode montage, stimulation dose, and differences in subject populations. In addition, these differences make combining the results of smaller studies more difficult, limiting the capacity for pooled estimate of their effect.

As studies continue to be published, there should be an increased focus on the existing consensus for tDCS research, ${ }^{88-90}$ and more specific parameters agreed for PD research. Consistent with this, all studies should provide clear methodology which details the specifics of the stimulation protocol and leaves no ambiguity or need for assumptions regarding testing conditions. We recommend that future studies provide, in addition to conventional scientific rigor (eg, randomization concealment, well defined outcomes, minimized bias), a detailed description of methods, including all parameters of stimulation, to enable experimental replication and allow for future modeling. There should be an increased focus on well-defined, robust protocols exploring session duration, repeated measures, and therapy as an adjunct to
tDCS. As some studies have demonstrated an interplay between tDCS and on/off states and more generally with PD pharmacological treatments, there is a need to define and quantify these clearly in parallel to the stimulation protocol, so that their interaction can be better understood.

\section{Disease severity}

Studies should also compare interventions between participant groups with varying severity of cognitive impairment, to provide insight into which stages of disease progression are most likely to benefit from cognitive training and brain stimulation.

A word of caution, however: there is a growing do-it-yourself tDCS community across the world, ${ }^{91}$ with a concerning expansion in uncontrolled use of tDCS outside clinical and academic settings. Trading companies contribute to a misguided development, promoting tDCS outside medical remits and instead for the purpose of self-neurological enhancement, to improve memory, attention, learning, decision-making, problem-solving, sleep, physical endurance, or gaming performance. ${ }^{92}$ Therefore, there is a real need to consider tDCS only in light of rigorous clinical studies that have been published in reputable peer-reviewed journals.

\section{Conclusion}

PD is a progressive neurodegenerative disorder characterized by motor and non-motor complications that arise from both the underlying disease process and in relation to medication use. While invasive treatments such as DBS have revolutionized the management of PD, such treatments carry significant anesthetic and surgical risks. Therefore, there is a growing need to translate the positive findings of non-invasive brain stimulation (particularly tDCS) studies upon motor and nonmotor PD symptoms into mainstream clinical practice. To do so, large-scale, multicenter, randomized, double-blind, Phase III studies using standardized protocols based on the more robust published pilot data should be encouraged, in preference to small-scale proof-of-principle pilot studies. Future tDCS studies in PD should therefore carefully consider the role of dopamine, disease severity, adjunctive physical or cognitive therapies, lateralization effects of tDCS, stimulation duration, current density, and electrode montages based on currently available evidence.

\section{Acknowledgment}

This research project was supported by the National Institute for Health Research University College London Hospitals Biomedical Research Centre. 


\section{Disclosure}

The authors report no conflicts of interest in this work.

\section{References}

1. Parrao-Diaz T, Chana-Cuevas P, Juri-Claverias C, Kunstmann C, TapiaNunez J. Evaluation of cognitive impairment in a population of patients with Parkinson's disease by means of the mini mental Parkinson test. Rev Neurol. 2004;40(6):339-344.

2. Jankovic J. Parkinson's disease: clinical features and diagnosis. J Neurol Neurosurg Psychiatry. 2008;79(4):368-376.

3. Chou YH, Hickey PT, Sundman M, Song AW, Chen NK. Effects of repetitive transcranial magnetic stimulation on motor symptoms in Parkinson disease: a systematic review and meta-analysis. JAMA Neurol. 2015;72(4):432-440.

4. Elahi B, Elahi B, Chen R. Effect of transcranial magnetic stimulation on Parkinson motor function - systematic review of controlled clinical trials. Mov Disord. 2009;24(3):357-363.

5. Nitsche MA, Paulus W. Excitability changes induced in the human motor cortex by weak transcranial direct current stimulation. J Physiol. 2000;527(3):633-639.

6. Fregni F, Boggio PS, Nitsche M, et al. Anodal transcranial direct current stimulation of prefrontal cortex enhances working memory. Exp Brain Res. 2005;166(1):23-30.

7. Nowak DA, Grefkes C, Ameli M, Fink GR. Interhemispheric competition after stroke: brain stimulation to enhance recovery of function of the affected hand. Neurorehabil Neural Repair. 2009;23(7): 641-656.

8. Lefaucheur JP. A comprehensive database of published tDCS clinical trials (2005-2016). Neurophysiol Clin. 2016;46(6):319-398.

9. Eggers C, Grüner U, Ameli M, Sarfeld AS, Nowak DA. 1Hz rTMS preconditioned by tDCS over the primary motor cortex in Parkinson's disease: absence of effect on arm lift and hand grip force control. Motor Control. 2012;16(2):284-292.

10. Grüner U, Eggers C, Ameli M, Sarfeld AS, Fink GR, Nowak DA. 1 $\mathrm{Hz}$ rTMS preconditioned by tDCS over the primary motor cortex in Parkinson's disease: effects on bradykinesia of arm and hand. J Neural Transm (Vienna). 2010;117(2):207-216.

11. von Papen M, Fisse M, Sarfeld AS, Fink GR, Nowak DA. The effects of $1 \mathrm{~Hz}$ rTMS preconditioned by tDCS on gait kinematics in Parkinson's disease. J Neural Transm (Vienna). 2014;121(7):743-754.

12. Bikson M, Grossman P, Thomas C, et al. Safety of transcranial direct current stimulation: evidence based update 2016. Brain Stimul. 2016;9(5):641-661.

13. Miranda PC, Lomarev M, Hallett M. Modeling the current distribution during transcranial direct current stimulation. Clin Neurophysiol. 2006;117(7):1623-1629.

14. Parazzini M, Fiocchi S, Rossi E, Paglialonga A, Ravazzani P. Transcranial direct current stimulation: estimation of the electric field and of the current density in an anatomical human head model. IEEE Trans Biomed Eng. 2011;58(6):1773-1780.

15. Braun R, Klein R, Walter HL, et al. Transcranial direct current stimulation accelerates recovery of function, induces neurogenesis and recruits oligodendrocyte precursors in a rat model of stroke. Exp Neurol. 2016;279:127-136.

16. Keuters MH, Aswendt M, Tennstaedt A, et al. Transcranial direct current stimulation promotes the mobility of engrafted NSCs in the rat brain. NMR Biomed. 2015;28(2):231-239.

17. Rueger MA, Keuters MH, Walberer M, et al. Multi-session transcranial direct current stimulation (tDCS) elicits inflammatory and regenerative processes in the rat brain. PLoS One. 2012;7(8):e43776.

18. Ruohonen J, Karhu J. tDCS possibly stimulates glial cells. Clin Neurophysiol. 2012;123(10):2006-2009.

19. Monai H, Ohkura M, Tanaka M, et al. Calcium imaging reveals glial involvement in transcranial direct current stimulation-induced plasticity in mouse brain. Nat Commun. 2016;7:11100.
20. Tanaka T, Takano Y, Tanaka S, et al. Transcranial direct-current stimulation increases extracellular dopamine levels in the rat striatum. Front Syst Neurosci. 2013;7:6.

21. Lu C, Wei Y, Hu R, Wang Y, Li K, Li X. Transcranial direct current stimulation ameliorates behavioral deficits and reduces oxidative stress in 1-methyl-4-phenyl-1, 2, 3, 6-tetrahydropyridine-induced mouse model of Parkinson's disease. Neuromodulation. 2015;18(6):442-447.

22. Li H, Lei X, Yan T, et al. The temporary and accumulated effects of transcranial direct current stimulation for the treatment of advanced Parkinson's disease monkeys. Sci Rep. 2015;5:12178.

23. Lefaucheur JP. Principles of therapeutic use of transcranial and epidural cortical stimulation. Clin Neurophysiol. 2008;119(10):2179-2184.

24. Lefaucheur JP. Methods of therapeutic cortical stimulation. Neurophysiol Clin. 2009;39(1):1-4.

25. Fregni F, Boggio PS, Santos MC, et al. Noninvasive cortical stimulation with transcranial direct current stimulation in Parkinson's disease. Mov Disord. 2006;21(10):1693-1702.

26. Benninger DH, Lomarev M, Lopez G, et al. Transcranial direct current stimulation for the treatment of Parkinson's disease. J Neurol Neurosurg Psychiatry. 2010;81(10):1105-1111.

27. Kuo MF, Paulus W, Nitsche MA. Boosting focally-induced brain plasticity by dopamine. Cereb Cortex. 2007;18(3):648-651.

28. Doruk D, Gray Z, Bravo GL, Pascual-Leone A, Fregni F. Effects of tDCS on executive function in Parkinson's disease. Neurosci Lett. 2014;582:27-31.

29. Costa-Ribeiro A, Maux A, Bosford T, et al. Transcranial direct current stimulation associated with gait training in Parkinson's disease: a pilot randomized clinical trial. Dev Neurorehabil. 2017;20(3):121-128.

30. Schabrun SM, Lamont RM, Brauer SG. Transcranial direct current stimulation to enhance dual-task gait training in Parkinson's disease: a pilot RCT. PLoS One. 2016;11(6):e0158497.

31. Salimpour Y, Mari ZK, Shadmehr R. Altering effort costs in Parkinson's disease with noninvasive cortical stimulation. $J$ Neurosci. 2015;35(35):12287-12302.

32. Morgante F, Espay AJ, Gunraj C, Lang AE, Chen R. Motor cortex plasticity in Parkinson's disease and levodopa-induced dyskinesias. Brain. 2006;129(4):1059-1069.

33. Ferrucci R, Cortese F, Bianchi M, et al. Cerebellar and motor cortical transcranial stimulation decrease levodopa-induced dyskinesias in Parkinson's disease. Cerebellum. 2016;15(1):43-47.

34. Schrag A, Jahanshahi M, Quinn N. What contributes to quality of life in patients with Parkinson9s disease? J Neurol Neurosurg Psychiatry. 2000;69(3):308-312.

35. Hely MA, Morris JG, Traficante R, Reid WG, O'Sullivan DJ, Williamson PM. The Sydney multicentre study of Parkinson's disease: progression and mortality at 10 years. J Neurol Neurosurg Psychiatry. 1999;67(3):300-307.

36. Hely MA, Reid WG, Adena MA, Halliday GM, Morris JG. The Sydney multicenter study of Parkinson's disease: the inevitability of dementia at 20 years. Mov Disord. 2008;23(6):837-844.

37. Debaere F, Wenderoth N, Sunaert S, Van Hecke P, Swinnen SP. Internal vs external generation of movements: differential neural pathways involved in bimanual coordination performed in the presence or absence of augmented visual feedback. Neuroimage. 2003;19(3):764-776.

38. Berardelli A, Rothwell JC, Thompson PD, Hallett M. Pathophysiology of bradykinesia in Parkinson's disease. Brain. 2001;124(11):2131-2146.

39. Samuel M, Ceballos-Baumann AO, Blin J, et al. Evidence for lateral premotor and parietal overactivity in Parkinson's disease during sequential and bimanual movements. a PET study. Brain. 1997;120(6):963-976.

40. Playford ED, Jenkins IH, Passingham RE, Nutt J, Frackowiak RS, Brooks DJ. Impaired mesial frontal and putamen activation in Parkinson's disease: a positron emission tomography study. Ann Neurol. 1992;32(2):151-161.

41. Verheyden G, Purdey J, Burnett M, Cole J, Ashburn A. Immediate effect of transcranial direct current stimulation on postural stability and functional mobility in Parkinson's disease. Mov Disord. 2013;28(14):2040-2041.

42. Cramer SC. Repairing the human brain after stroke: I. Mechanisms of spontaneous recovery. Ann Neurol. 2008;63(3):272-287. 
43. Galletly R, Brauer SG. Does the type of concurrent task affect preferred and cued gait in people with Parkinson's disease? Aust J Physiother. 2005;51(3):175-180.

44. Nieuwboer A, Kwakkel G, Rochester L, et al. Cueing training in the home improves gait-related mobility in Parkinson's disease: the RESCUE trial. J Neurol Neurosurg Psychiatry. 2007;78(2):134-140.

45. Lim I, van Wegen E, Jones D, et al. Does cueing training improve physical activity in patients with Parkinson's disease? Neurorehabil Neural Repair. 2010;24(5):469-477.

46. Lee SJ, Yoo JY, Ryu JS, Park HK, Chung SJ. The effects of visual and auditory cues on freezing of gait in patients with Parkinson disease. Am J Phys Med Rehabil. 2012;91(1):2-11.

47. Nieuwboer A. Cueing for freezing of gait in patients with Parkinson's disease: a rehabilitation perspective. Mov Disord. 2008;23(Suppl 2):S475-S481.

48. Del Olmo MF, Cheeran B, Koch G, Rothwell JC. Role of the cerebellum in externally paced rhythmic finger movements. $J$ Neurophysiol. 2007;98(1):145-152.

49. Costa-Ribeiro A, Maux A, Bosford T, et al. Dopamine-independent effects of combining transcranial direct current stimulation with cued gait training on cortical excitability and functional mobility in Parkinson's disease. J Rehabil Med. 2016;48(9):819-823.

50. Kaski D, Dominguez RO, Allum JH, Islam AF, Bronstein AM. Combining physical training with transcranial direct current stimulation to improve gait in Parkinson's disease: a pilot randomized controlled study. Clin Rehabil. 2014;28(11):1115-1124.

51. Lattari E, Costa SS, Campos C, de Oliveira AJ, Machado S, Neto GA. Can transcranial direct current stimulation on the dorsolateral prefrontal cortex improves balance and functional mobility in Parkinson's disease? Neurosci Lett. 2017;636:165-169.

52. Manenti R, Brambilla M, Benussi A, et al. Mild cognitive impairment in Parkinson's disease is improved by transcranial direct current stimulation combined with physical therapy. Mov Disord. 2016;31(5):715-724.

53. Biundo R, Weis L, Fiorenzato E, et al. Double-blind randomized trial of t-DCS versus sham in Parkinson patients with mild cognitive impairment receiving cognitive training. Brain Stimul. 2015;8(6):1223-1225.

54. Manenti R, Brambilla M, Rosini S, et al. Time up and go task performance improves after transcranial direct current stimulation in patient affected by Parkinson's disease. Neurosci Lett. 2014;580:74-77.

55. Swank C, Mehta J, Criminger C. Transcranial direct current stimulation lessens dual task cost in people with Parkinson's disease. Neurosci Lett. 2016;626:1-5.

56. Valentino F, Cosentino G, Brighina F, et al. Transcranial direct current stimulation for treatment of freezing of gait: a cross-over study. Mov Disord. 2014;29(8):1064-1069.

57. Nieoullon A, Cheramy A, Glowinski J. Release of dopamine evoked by electrical stimulation of the motor and visual areas of the cerebral cortex in both caudate nuclei and in the substantia nigra in the cat. Brain Res. 1978;145(1):69-83.

58. Whitton PS. Glutamatergic control over brain dopamine release in vivo and in vitro. Neurosci Biobehav Rev. 1997;21(4):481-488.

59. Polanía R, Paulus W, Nitsche MA. Modulating cortico-striatal and thalamo-cortical functional connectivity with transcranial direct current stimulation. Hum Brain Mapp. 2012;33(10):2499-2508.

60. Goldman-Rakic PS. The cortical dopamine system: role in memory and cognition. Adv Pharmacol. 1997;42:707-711.

61. Kulisevsky J. Role of dopamine in learning and memory. Drugs Aging. 2000;16(5):365-379.

62. Lees AJ, Smith E. Cognitive deficits in the early stages of Parkinson's disease. Brain. 1983;106(2):257-270.

63. Taylor AE, Saint-Cyr JA, Lang AE. Frontal lobe dysfunction in Parkinson's disease: the cortical focus of neostriatal outflow. Brain. 1986;109(5):845-883.

64. Grimm S, Beck J, Schuepbach D, et al. Imbalance between left and right dorsolateral prefrontal cortex in major depression is linked to negative emotional judgment: an fMRI study in severe major depressive disorder. Biol Psychiatry. 2008;63(4):369-376.
65. Fregni F, Boggio PS, Nitsche M, et al. Anodal transcranial direct current stimulation of prefrontal cortex enhances working memory. Exp Brain Res. 2005;166(1):23-30.

66. Boggio PS, Rigonatti SP, Ribeiro RB, et al. A randomized, doubleblind clinical trial on the efficacy of cortical direct current stimulation for the treatment of major depression. Int $J$ Neuropsychopharmacol. 2008;11(2):249-254.

67. Fregni F, Boggio PS, Nitsche MA, Marcolin MA, Rigonatti SP, PascualLeone A. Treatment of major depression with transcranial direct current stimulation. Bipolar Disord. 2006;8(2):203-204.

68. Boggio PS, Fregni F, Bermpohl F, et al. Effect of repetitive TMS and fluoxetine on cognitive function in patients with Parkinson's disease and concurrent depression. Mov Disord. 2005;20(9):1178-1184.

69. Fregni F, Santos CM, Myczkowski ML, et al. Repetitive transcranial magnetic stimulation is as effective as fluoxetine in the treatment of depression in patients with Parkinson's disease. J Neurol Neurosurg Psychiatry. 2004;75(8):1171-1174.

70. Wu AD, Fregni F, Simon DK, Deblieck C, Pascual-Leone A. Noninvasive brain stimulation for Parkinson's disease and dystonia. Neurotherapeutics. 2008;5(2):345-361.

71. Boggio PS, Ferrucci R, Rigonatti SP, et al. Effects of transcranial direct current stimulation on working memory in patients with Parkinson's disease. J Neurol Sci. 2006;249(1):31-38.

72. Mottaghy FM, Krause BJ, Kemna LJ, et al. Modulation of the neuronal circuitry subserving working memory in healthy human subjects by repetitive transcranial magnetic stimulation. Neurosci Lett. 2000;280(3):167-170.

73. Mull BR, Seyal M. Transcranial magnetic stimulation of left prefrontal cortex impairs working memory. Clin Neurophysiol. 2001;112(9):1672-1675.

74. Fregni F, Marcolin MA, Myczkowski M, et al. Predictors of antidepressant response in clinical trials of transcranial magnetic stimulation. Int J Neuropsychopharmacol. 2006;9(6):641-654.

75. Iyer MB, Mattu U, Grafman J, Lomarev M, Sato S, Wassermann EM. Safety and cognitive effect of frontal DC brain polarization in healthy individuals. Neurology. 2005;64(5):872-875.

76. Pereira JB, Junqué C, Bartrés-Faz D, et al. Modulation of verbal fluency networks by transcranial direct current stimulation (tDCS) in Parkinson's disease. Brain Stimul. 2013;6(1):16-24.

77. van Eimeren T, Monchi O, Ballanger B, Strafella AP. Dysfunction of the default mode network in Parkinson disease: a functional magnetic resonance imaging study. Arch Neurol. 2009;66(7):877-883.

78. Ibarretxe-Bilbao N, Zarei M, Junque C, et al. Dysfunctions of cerebral networks precede recognition memory deficits in early Parkinson's disease. Neuroimage. 2011;57(2):589-597.

79. Keeser D, Padberg F, Reisinger E, et al. Prefrontal direct current stimulation modulates resting EEG and event-related potentials in healthy subjects: a standardized low resolution tomography (sLORETA) study. Neuroimage. 2011;55(2):644-657.

80. Merzagora AC, Foffani G, Panyavin I, et al. Prefrontal hemodynamic changes produced by anodal direct current stimulation. Neuroimage. 2010;49(3):2304-2310.

81. Yarnall AJ, Breen DP, Duncan GW, et al. Characterizing mild cognitive impairment in incident Parkinson disease: the ICICLE-PD study. Neurology. 2014;82(4):308-316.

82. Forogh B, Rafiei M, Arbabi A, Motamed MR, Madani SP, Sajadi S. Repeated sessions of transcranial direct current stimulation evaluation on fatigue and daytime sleepiness in Parkinson's disease. Neurol Sci. 2017;38(2):249-254.

83. Loo CK, Alonzo A, Martin D, Mitchell PB, Galvez V, Sachdev P. Transcranial direct current stimulation for depression: 3-week, randomised, sham-controlled trial. Br J Psychiatry. 2012;200(1):52-59.

84. Carlfjord S, Lindberg M, Bendtsen P, Nilsen P, Andersson A. Key factors influencing adoption of an innovation in primary health care: a qualitative study based on implementation theory. BMC Family Practice. 2010;11(1):60. 
85. Fritsch B, Reis J, Martinowich K, et al. Direct current stimulation promotes BDNF-dependent synaptic plasticity: potential implications for motor learning. Neuron. 2010;66(2):198-204.

86. Gasparoli E, Delibori D, Polesello G, et al. Clinical predictors in Parkinson's disease. Neurol Sci. 2002;23:s77-s78.

87. Huber SJ, Miller H, Bohaska L, Christy JA, Bornstein RA. Asymmetrical cognitive differences associated with hemiparkinsonism. Arch Clin Neuropsychol. 1992;7(6):471-480.

88. Buch ER, Santarnecchi E, Antal A, et al. Effects of tDCS on motor learning and memory formation: a consensus and critical position paper. Clin Neurophysiol. 2017;128(4):589-603.
89. Brunoni AR, Nitsche MA, Bolognini N, et al. Clinical research with transcranial direct current stimulation (tDCS): challenges and future directions. Brain Stimul. 2012;5(3):175-195.

90. Lefaucheur JP, Antal A, Ayache SS, et al. Evidence-based guidelines on the therapeutic use of transcranial direct current stimulation (tDCS). Clin Neurophysiol. 2017;128(1):56-92.

91. Smith CD. The electric brain: do-it-yourself healthcare with transcranial direct current stimulation. J Responsible Innovation. 2017;1(1):1-12.

92. Hamilton R, Messing S, Chatterjee A. Rethinking the thinking cap ethics of neural enhancement using noninvasive brain stimulation. Neurology. 2011;76(2):187-193.
Journal of Parkinsonism and Restless Legs Syndrome

\section{Publish your work in this journal}

Journal of Parkinsonism and Restless Legs Syndrome is an online, open access, peer-reviewed journal. The journal publishes review articles, historical reviews, original research articles, case reports, letters to the editor, clinical teaching cases, neuroradiology highlights, neuropathology highlights, neuropsychiatry highlights, autobiographies, conference

\section{Dovepress}

proceedings, abstracts and book reviews. The manuscript management system is completely online and includes a very quick and fair peerreview system, which is all easy to use. Visit http://www.dovepress.com testimonials.php to read real quotes from published authors.

Submit your manuscript here: https://www.dovepress.com/journal-of-parkinsonism-and-restless-legs-syndrome-journal 